Water Films, Asphaltenes, and Wettability Alteration

R. Kaminsky and C.J. Radke

Earth Science Division of the Lawrence Berkeley National Laboratory and Department of Chemical Engineering

University of California

Berkeley, CA 94720

$+$

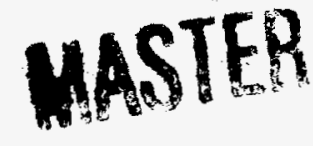

$j^{p}$

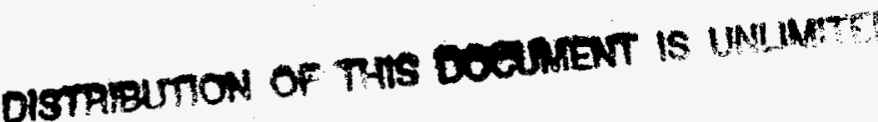




\section{DISCLAIMER}

This report was prepared as an account of work sponsored by an agency of the United States Government. Neither the United States Government nor any agency thereof, nor any of their employees, makes any warranty, express or implied, or assumes any legal liability or responsibility for the accuracy, completeness, or usefulness of any information, apparatus, product, or process disclosed, or represents that its use would not infringe privately owned rights. Reference herein to any specific commercial product, process, or service by trade name, trademark, manufacturer, or otherwise does not necessarily constitute or imply its endorsement, recommendation, or favoring by the United States Government or any agency thereof. The views and opinions of authors expressed herein do not necessarily state or reflect those of the United States Government or any agency thereof. 


\section{DISCLAIMER}

Portions of this document may be illegible electronic image products. Images are produced from the best available original document. 


\title{
Water Films, Asphaltenes, and Wettability Alteration
}

\author{
R. Kaminsky and C.J. Radke \\ Earth Science Division of the Lawrence Berkeley National Laboratory \\ and Department of Chemical Engineering \\ University of California \\ Berkeley, CA 94720
}

\section{RECEIVED \\ JUN 021998 \\ OSTI}

\begin{abstract}
We present a transport model for asphaltene diffusion from an oil/water interface through a water film followed by adsorption at a solid/water interface. Using a Langmuir adsorption isotherm, the effect of asphaltene aqueous solubility and adsorption constant $K$ on equilibration time are established. For $K$ greater than $1 \mathrm{~nm}$ and asphaltene solubilities down to $0.1 \mathrm{ppb}$, adsorption equilibrium, taken to be $1 \mathrm{mg} / \mathrm{m}^{2}$, occurs within a few hours. Negligible asphaltene solubility does not explain why a water film prevents asphaltene adsorption and wettability alteration in reservoir rock.
\end{abstract}

\section{Introduction}

One of the fundamental pieces of information required for efficient design of oil recovery processes is the reservoir wettability, loosely defined as the preferential affinity of the solid matrix for either the aqueous or oil phases. Current wettability research is aimed at envisioning and understanding reservoir wettability and its development, and varies from microscopic to reservoir scales ${ }^{1-6}$. Underlying this work are three basic assumptions that are now widely accepted as near fact. The first and most significant of these was discovered by Salathiel in his landmark 1972 study ${ }^{7}$. Counter to previous opinion of universal water-wet reservoirs, Salathiel hypothesized a mixed-wet condition with large pores being oil-wet and smaller pores being water-wet, and with the oil-wet and water-wet regions continuously connected. As shown in his core flood experiments, mixed-wet rock exhibited very low residual oil saturations but slow oil production rates at these low saturations. The second assumption, consistent with Salathiel's vision of continuous oil and water phases, is that configurations of oil in pores involve either direct contact between oil and rock, or separation of the oil phase from the solid by aqueous films, as illustrated in Fig. 1. Existence of such water films was first postulated for Athabasca tar sands in the 1930 ' $\mathrm{s}^{8}$, and since then much has been done to both visualize ${ }^{6}$ and describe ${ }^{9,10}$ them. The third basic assumption is that in a given pore, when a critical capillary pressure is exceeded, water films destabilize and rupture to an adsorbed molecular film of up to several water monolayers. Crude oil now contacts rock directly, allowing polar oil species to adsorb and/or deposit onto the rock. It is this process that locally reverses the wettability of the rock from water-wet to oil-wet ${ }^{11}$. Specifically, asphaltenes, a group of oil components defined by their solubility behavior, are envisioned to be responsible for the wettability alteration because of their polar functional groups which may interact with mineral oxide surfaces. The role of the water films is thus absolutely essential: presence of a thick water film between the crude oil and the rock surface prevents contact of asphaltenes with the rock and accordingly protects against wettability reversal ${ }^{10}$.

Evidence is diverse for the strong role of asphaltenes in influencing reservoir wettability. In imbibition tests of sandstone and limestone cores treated with different oil fractions, oil-wet cores are produced only by the heaviest fraction, the residue ${ }^{12}$, which contains the asphaltenes. In Salathiel's experiments ${ }^{7}$, asphaltic oil produced mixed-wet cores. More recent experiments show strong adsorption of asphaltenes from organic solutions onto clays and sandstones, causing 
dramatic wettability shifts ${ }^{13,14}$. Finally, upon contacting deasphalted crude oil with smooth glass surfaces, Buckley and Morrow found no wettability alteration, as contrasted with strong wettability alteration caused by the original oil ${ }^{1}$. These results contribute to the belief that asphaltenes cause reservoir mixed wettability.

Much is still unknown about the mechanism of wettability alteration. The accepted explanation of the water film's protective role is that the virtual insolubility of asphaltenes in water precludes their transport to the rock surfaces. (Otherwise, initially water-wet reservoirs should eventually become completely oil wet upon initial migration and invasion of asphaltic crude oil.) Over geologic time, however, even a minuscule water solubility of asphaltenes permits diffusion through a water layer and adsorption at the rock surface. Unfortunately, experimental study of this adsorption process is hindered by the chameleon-like behavior of asphaltenes: their structure is strongly dependent on solvent characteristics.

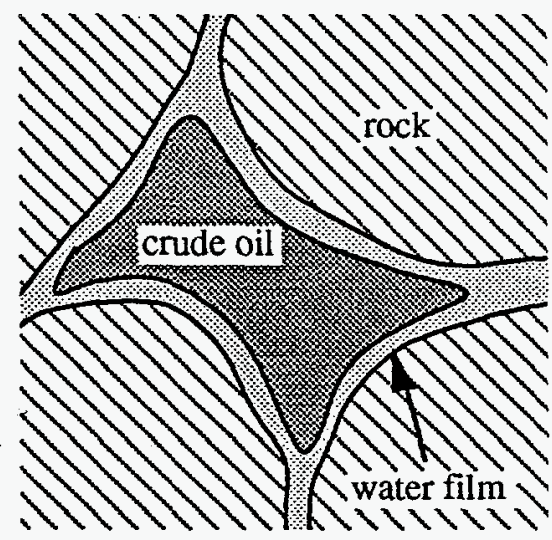

(a)

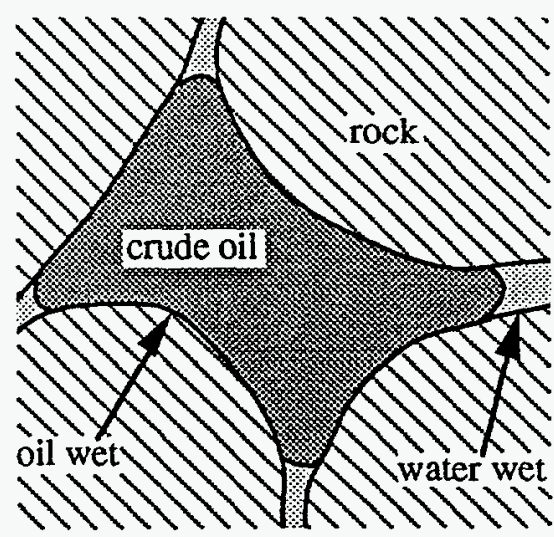

(b)

Fig. 1-Schematic of a pore cross-section in a porous medium. (a) Water-wet rock grains are surrounded by a thin film of brine and are not contacted by oil. (b) Mixed-wet pore has adjacent oil-wet and water-wet regions.

Asphaltenes are known to be large, heterocyclic aromatic compounds with nitrogen, oxygen, and sulfur atoms throughout the ring structure ${ }^{15}$. They are by definition insoluble int small alkanes, but soluble to different degrees in aromatic solvents. In crude oil, asphaltenes are believed to exist as aggregates of various sizes stabilized by an outer coating of amphiphilic resin molecules, which contain a polar end directed toward the asphaltenes, and a nonpolar aliphatic end which allows for solubility in the crude oil ${ }^{13,14,16,17}$. Adsorption of asphaltenes from organic solutions onto mineral surfaces displays a Langmuir adsorption isotherm and reversible adsorption at low asphaltene concentrations ${ }^{14,16,17}$. Higher concentrations lead to irreversible adsorption, possibly because asphaltene aggregation at the surface makes simultaneous bond breakage, a highly improbable event, necessary for desorption. When crude oil directly contacts a surface in the absence of water, initial adsorption and wettability alteration occurs immediately. The final configuration, after exchange of different molecular-weight asphaltenes, may take days or longer to be completed, but results only in qualitatively different wettability ${ }^{18}$. It is dangerous to extrapolate these results to adsorption from an aqueous phase because asphaltene structure in water may only be speculated.

We address here the question of whether very low solubilities of asphaltenes in water can indeed prevent diffusion through a water film. The purpose of this paper is to calculate the total time for diffusion of low solubility asphaltenes 
through a water layer followed by adsorption onto the rock surface. This will in part confirm or deny the validity of the negligible solubility argument to explain the proposed protective role of water films in wettability alteration of reservoirs.

\section{Theory}

The problem we address is shown schematically in Fig. 2. An oil phase is separated from the rock by a water layer of thickness $\delta$. For connate water saturations typical of reservoir discovery, we take $\delta$ to be $100 \mathrm{~nm}$ or less, and certainly less than $1 \mu \mathrm{m}$. At the oil/water interface, a constant equilibrium aqueous-phase solubility concentration of asphaltenes is assumed. At the solid/aqueous interface, the rate of asphaltene adsorption is identical to the diffusive flux of asphaltenes to the surface. Adsorption density, $\Gamma$, is assumed to follow a simple Langmuir isotherm, and local equilibrium is assumed at the solid/water interface. Because our primary concern is the role of asphaltene diffusion through the water layer, kinetic resistance to adsorption is not considered. Clearly, including finite kinetics increases the adsorption time. However, we argue that dramatic wettability reversals occur through early contact of oil components with the surface. Subsequent rearrangement of the adsorbed molecules likely contributes secondary wettability effects. At zero initial time, the aqueous phase contains no asphaltenes, after which they begin to diffuse from the oil/water interface and adsorb upon arrival at the solid/water interface. Eventually, the solid surface equilibrates with adsorbed asphaltenes. Our goal is to investigate the effect of very low asphaltene solubility on the time to reach surface equilibration.

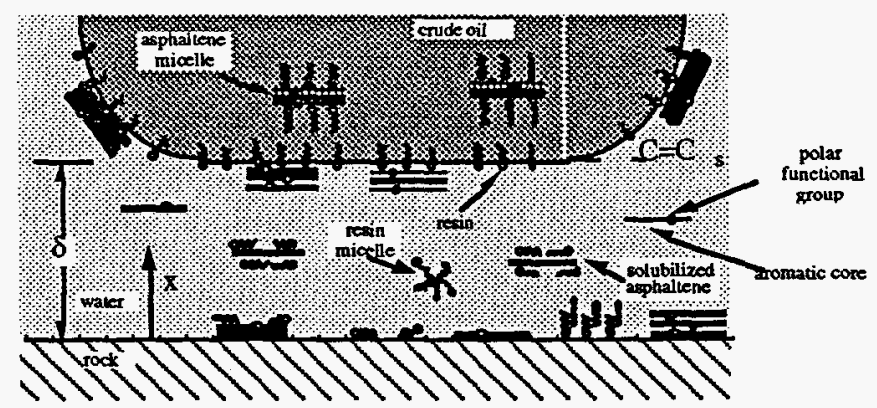

Fig. 2-Diffusion of asphaltenes and/or resins from the oil phase through a thin aqueous film followed by adsorption on the solid matrix.

Fig. 2 also illustrates potential configurations of asphaltenes and resins at the solid/water and oil/water interfaces and in the bulk oil and water phases. A single asphaltene molecule is drawn was a dark line with a circular patch representing the aromatic sheet with interspersed polar regions ${ }^{19}$. Within the crude oil, asphaltenes can exist as micelles surrounded by resin molecules, which are illustrated by a polar head group and hydrocarbon tail. We illustrate these micelles schematically by grouping several asphaltene sheets. Recent work ${ }^{19,20}$ on water-in-crude oil emulsions suggests that asphaltenes and resins adsorb at the oil/water interface as micelles of various size, depending upon solvent aromaticity and resin-to-asphaltene ratio. At the oil/water interface, the polar asphaltenes presumably lose their outer resin shell when oriented toward the water phase, as shown schematically in Fig. 2. It seems unlikely that resin-stabilized hydrophobic asphaltene micelles diffuse through the water phase and adsorb onto the surface. More likely is diffusion of smaller components: single, polar asphaltene molecules; resin molecules, which are likely more soluble than the asphaltene molecules because of their amphiphilicity and their lower molecular weight; inverted hydrophilic resin micelles, with alkyl stabilizing resin molecules oriented toward the core, with or without asphaltenes inside; or some small association of complexed asphaltenes and resins. These possible molecular structures are all displayed in Fig. 2. Asphaltenes are shown adsorbed at the solid/water interface with the planar faces toward the solid. Adsorption of asphaltenes probably occurs through polar interactions 
between the mineral surface and the heteroatoms or amine and carboxylic acid groups of the asphaltenes. Resins may also adsorb through polar interactions, possibly forming bilayers at high enough concentrations. When enough molecules have reached the surface, adsorption likely becomes irreversible after rearrangement and aggregation from an initial configuration. We treat the diffusion and adsorption process in Fig. 2 as occurring via a single component, denoted generically as an asphaltene molecule. This viewpoint is clearly over-simplified, but should nevertheless allow an estimation of the expected time scale of the process.

Diffusion of surface-active species to an interface followed by adsorption is a well-studied problem in the dynamic surface tension literature ${ }^{21}$. The classical results of both Ward and Tordai ${ }^{22}$ and Sutherland ${ }^{23}$ are similar to the asphaltene adsorption problem, in that all three demand solution of Fick's law of diffusion. We, however, are interested in diffusion through a finite thickness film initially devoid of the diffusing species, whereas they consider diffusion from a bulk solution, initially at a finite constant concentration which is fixed infinitely far from the interface.

The asphaltene diffusion problem in nondimensional variables is stated as follows:

$$
\frac{\partial C(z, \tau)}{\partial \tau}=\frac{\partial^{2} C(z, \tau)}{\partial z^{2}}
$$

with boundary conditions:

$$
\begin{aligned}
& C(z, 0)=0 \\
& C(1, \tau)=c_{s} \delta / \Gamma_{\max } \equiv C_{s} \\
& \frac{K}{\delta} \frac{\partial \theta(\tau)}{\partial \tau}=\left.\frac{\partial C(z, \tau)}{\partial z}\right|_{z=0}
\end{aligned}
$$

and with a Langmuir isotherm:

$$
\theta(\tau)=\frac{\frac{K}{\delta} C(0, \tau)}{1+\frac{K}{\delta} C(0, \tau)}
$$

where $C=c \delta \Gamma_{\max }, \theta=\Gamma / \Gamma_{\max }, z=x / \delta, \tau=D t / \delta^{2}, x$ is the distance fram the rock surface, $t$ is time, $K$ is the adsorption equilibrium constant in the Langmuir isotherm, $c$ is the asphaltene aqueous-phase concentration, $c_{s}$ is the solubility concentration, $\Gamma$ is the asphaltene adsorption at the solid/water interface, $\Gamma_{\max }$ corresponds to maximum adsorption, and $D$ is the asphaltene diffusivity in water. $K$ gives an indication of the thickness of the asphaltene adsorbed layer in the linear region of the isotherm.

As indicated above, the transient asphaltene concentration profile, $C(z, \tau)$, depends upon two dimensionless parameters, $K / \delta$ and $C_{S}=c_{S} \delta / \Gamma_{\max }$. The first of these parameters gives the ratio of the adsorption layer thickness to the diffusion path length, the water film thickness. The second parameter is a capacity factor, indicating the relative asphaltene capacity of the bulk to that of the surface: $c_{S} \delta \Gamma_{\max }=c_{\mathcal{S}} / \Gamma_{\max } a_{\nu}$, where $a_{v}$ is the rock surface area to aqueous film volume ratio. The product of the two parameters, $c_{S} K / \Gamma_{\max }$, is the ratio of the solubility concentration to the characteristic concentration at which maximum adsorption is reached, $\Gamma_{\max } / K$. 
Solution to Eqs. 1 - 5 is obtained by writing:

$$
C(z, \tau)=U(z, \tau)+V(z, \tau)
$$

with boundary conditions on $U$ :

$$
\text { 1. } U(z, 0)=0
$$

2. $U(0, \tau)=0$

$$
\text { 3. } U(1, \tau)=C_{s}
$$

$U$ is easily found to be the series solution:

$$
U(z, \tau)=C_{s}\left[z+\frac{2}{\pi} \sum_{n=1}^{\infty} \frac{(-1)^{n}}{n} \sin (n \pi z) e^{-n^{2} \pi^{2} \tau}\right]
$$

Solving for $V$ is not straightforward because of the time-dependent boundary condition at the solid interface, Eq. 4. $V(z, \tau)$ is, however, amenable to solution by Duhamel's superposition integral ${ }^{24}$ :

$$
V(z, \tau)=\int_{0}^{\tau} \frac{\partial V(0, \zeta)}{\partial \zeta}\left[(1-z)-\frac{2}{\pi} \sum_{n=1}^{\infty} \frac{1}{n} \sin (n \pi z) e^{-n^{2} \pi^{2}(\tau-\zeta)}\right] \mathrm{d} \zeta
$$

In order to find $V(0, \tau)$, the solid/water boundary condition in Eq. 4 is applied, leading to the following result, implicit in $V(0, \tau):$

$$
\frac{K}{\delta} \frac{\partial V(0, \tau)}{\partial \tau}=\left\{C_{s}\left[1+2 \sum_{n=1}^{\infty}(-1)^{n} e^{-n^{2} \pi^{2} \tau}\right]-\int_{0}^{\tau}\left[\frac{\partial V(0, \zeta)}{\partial \zeta}\left(1+2 \sum_{n=1}^{\infty} e^{-n^{2} \pi^{2}(\tau-\zeta)}\right)\right] \mathrm{d} \zeta\right\} \times\left[1+\frac{K}{\delta} V(0, \tau)\right]^{2}
$$

This equation is solved numerically by discretizing the integral and derivatives over equal sized time steps, defined as $j \Delta \tau$ $=\tau_{j+1}$, and then solving for $V\left(0, \tau_{m+1}\right)$ in terms of $V\left(0, \tau_{j \leq m}\right)$. We use a fully implicit scheme: the right side of Eq. 10 is evaluated at $\tau_{m+1}$ and the derivative as $\left[V\left(0, \tau_{m+1}\right)-V\left(0, \tau_{m}\right)\right] / \Delta \tau$. This yields a cubic equation for each $V\left(0, \tau_{m+1}\right)$ :

$$
a_{3}\left[V\left(0, \tau_{m+1}\right)\right]^{3}+a_{2}\left[V\left(0, \tau_{m+1}\right)\right]^{2}+a_{1} V\left(0, \tau_{m+1}\right)+a_{0}=0
$$

where

$$
\begin{aligned}
& a_{3}=\Delta \tau+\frac{2}{\pi^{2}} \sum_{n=1}^{\infty} \frac{1-e^{-n^{2} \pi^{2} \Delta \tau}}{n^{2}} \\
& a_{2}\left(V\left(0, \tau_{m}\right)\right)=2 \frac{\delta}{K} a_{3}-g\left(V\left(0, \tau_{m}\right)\right) \\
& a_{1}\left(V\left(0, \tau_{m}\right)\right)=\frac{\delta}{K}\left[1+\frac{\delta}{K} a_{3}-2 g\left(V\left(0, \tau_{m}\right)\right)\right]
\end{aligned}
$$




$$
\begin{aligned}
& a_{0}\left(V\left(0, \tau_{m}\right)\right)=-\frac{\delta}{K}\left[\frac{\delta}{K} g\left(V\left(0, \tau_{m}\right)\right)+V\left(0, \tau_{m}\right)\right] \\
& g\left(V\left(0, \tau_{m}\right)\right)=C_{s}\left[\Delta \tau+2 \Delta \tau \sum_{n=1}^{\infty}(-1)^{n} e^{-n^{2} \pi^{2} m \Delta \tau}\right]+V\left(0, \tau_{m+1}\right) a_{3}- \\
& \sum_{j=1}^{m-1}\left\{\left[V\left(0, \tau_{j+1}\right)-V\left(0, \tau_{j}\right)\right] \times\left[\Delta \tau+\frac{2}{\pi^{2}} \sum_{n=1}^{\infty} \frac{e^{-n^{2} \pi^{2} m \Delta \tau}}{n^{2}}\left(e^{n^{2} \pi^{2} j \Delta \tau}-e^{n^{2} \pi^{2}(j-1) \Delta \tau}\right)\right]\right\}
\end{aligned}
$$

which is solved using the Newton-Raphson method. Typical time increments are $\Delta \tau=10^{-3}$. If required, the full solution $C(z, \tau)$ can be constructed. To establish the time to reach adsorption equilibrium, we are interested in the behavior at the solid surface.

\section{Results and Discussion}

We desire the time at which adsorption equilibrium is reached, $\tau_{e q}$, as a function of both $K J \delta$ and $C_{S}$. $\tau_{e q}$ is defined here as the time at which $\theta$ reaches 0.99 of its maximum attainable value, $\theta\left(C_{S}\right)$, which is in general less than unity. Graphs of $\tau_{e q}$ versus $K / \delta$ for different values of $C_{S}$, and $\tau_{e q}$ versus $C_{S}$ for different values of $K / \delta$ are shown on $\log -\log$ scales in Figs. 3 and 4, respectively. Fig. 3 shows a clear maximum at roughly $K / \delta=C_{S}{ }^{-1}$. This is the location of the elbow in the adsorption isotherm. For values of $K / \delta<C_{S}^{-1}$, the Langmuir isotherm equilibration times (solid lines) approximate those of a linear isotherm, $\theta=(K / \delta) C$. The linear isotherm solution, shown by the dashed line in Fig. 3, is given in Carslaw and Jaeger 25 , and predicts that the adsorption equilibration time varies linearly with $K / \delta$.

$$
\tau_{e q}=\tau_{o}(\alpha)+\frac{K}{\delta} \ln \alpha^{-1} \quad \text { for } K / \delta<C_{s}^{-1}
$$

where we have chosen to define equilibrium by $\alpha=0.01$ (i.e. $\Gamma / \Gamma_{e q}=0.99$ ), and both the intercept $\tau_{0}$ and the slope depend on $\alpha$. At large $K / \delta$ the effect of increasing $K / \delta$ in the linear isotherm regime is to increase linearly the amount of adsorption, and therefore the time to reach this increasing equilibrium amount. At very small $K / \delta$ the time to reach equilibrium approaches the constant $\tau_{0}$. To find $\tau_{0}$, we calculate the building solute concentration at the interface $C(0, \tau)$ for a zero flux boundary condition. $\Gamma$ then follows from Eq. 5 .

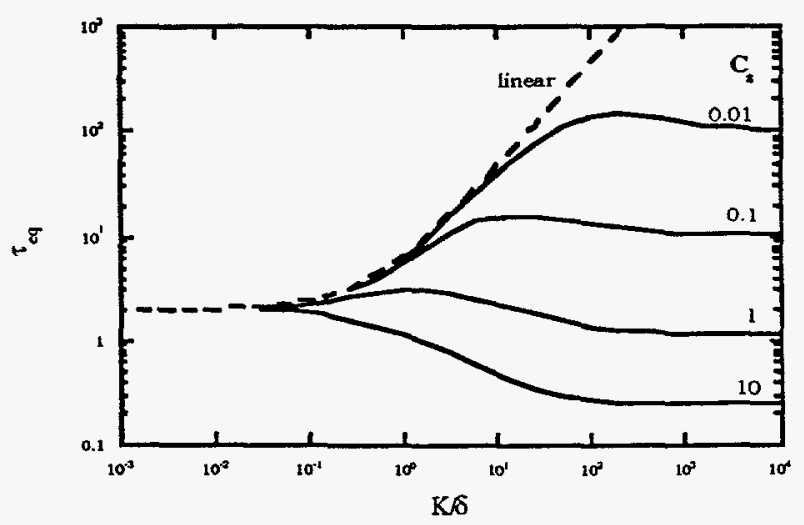


Fig. 3-Adsorption equilibration times as a function of $K / \delta$ for varying $C_{S}$ and for the linear isotherm ( $C_{S} \rightarrow 0$ ), shown as a dashed line.

For $K / \delta<C_{S}^{-1}$ in Fig. 3, all Langmuir adsorption equilibrium times approach the linear isotherm. This is seen in Fig. 3 where the solid lines merge into the dashed line.

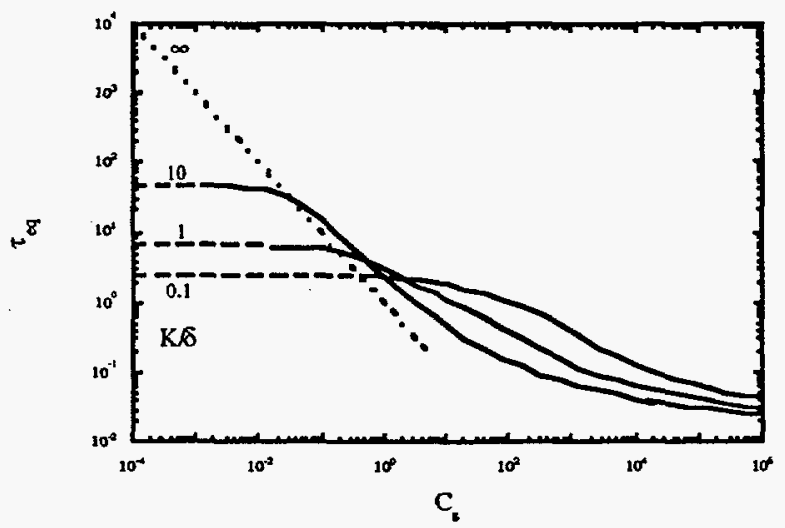

Fig. 4-Adsorption equilibration times as a function of $c_{S}$ for varying $K \delta$. Daşhed lines correspond to the $c_{S} \rightarrow 0$ limit of a linear isotherm whereas the dotted line corresponds to the $K \delta>C_{s}^{-1} \rightarrow \infty$ limit of Eq. 16 .

In the second regime of Fig. 3, $K / \delta>C_{S}{ }^{-1}$, equilibrium time decreases with increasing $K / \delta$, and approaches an asymptotic value that depends on $C_{S}$. In the $K / \delta$ approaches infinity limit, adsorption at the solid surface saturates to $\Gamma_{\max }$ while the aqueous concentration adjacent to the solid remains essentially zero. The process can be thought of as having two distinct time steps: diffusion and saturation of adsorption to $\theta=1$, while zero aqueous-phase bulk concentration is maintained at the solid surface, followed by saturation of the bulk film to concentration $c_{s}$ with a no-flux boundary condition at the solid surface. Only the first time scale is relevant to our definition of $\tau_{\text {eq. }}$. To find this value of $\tau_{\mathrm{eq}}$, Eq. 4 is solved in the following form:

$$
\theta=\int_{0}^{\tau} \frac{\partial C}{\partial z}(0, \tau) \mathrm{d} \tau=1-\alpha \quad \text { for } K / \delta>C_{s}^{-1} \rightarrow \infty
$$

where $C(z, \tau)$ is now the solution to Eq. 1 with the solid/water interface boundary condition set to $C(0, \tau)=0$. We find that:

$$
\begin{gathered}
C_{s}\left[\tau_{e q}+2 \sum_{n=1}^{\infty} \frac{(-1)^{n+1}}{n^{2} \pi^{2}}\left(e^{-n^{2} \pi^{2} \tau_{e q}}-1\right)\right]=1-\alpha \\
\text { for } K / \delta>C_{s}^{-1} \rightarrow \infty
\end{gathered}
$$

For large values of $\tau_{e q}$, Eq. 15 reduces to the result

$$
\tau_{e q}=(1-\alpha) C_{s}^{-1} \quad \text { for } K / \delta>C_{s}^{-1} \rightarrow \infty
$$

Fig. 3 confirms this behavior for large $K / \delta$ Away from this asymptotic limit, equilibration of the bulk solution and equilibration of the interface occur together, thereby slowing the adsorption process and giving rise to the observed maxima. 
The same information can be plotted as $\tau_{e q}$ versus $C_{S}$, as shown in Fig. 4, allowing for examination of a larger range in $C_{S}$. This plot shows even more dramatically the effect of asphaltene aqueous solubility. Below a given $C_{S}$ value, which depends on $K / \delta$, a linear isotherm emerges, with equilibration time independent of solubility and linearly related to $K / \delta$, as quantified in Eq. 13. These curves correspond to the linear isotherm curve of Fig. 3 and, therefore, are also shown in Fig. 4 by dashed lines. Included as a dotted line in Fig. 4 is the infinite $K / \delta$ limit given by Eq. 16, which is valid only for $K / \delta>$ $C_{S}{ }^{-1}$. Once $C_{S}$ drops below $(K / \delta)^{-1}$, the equilibrium time approaches a finite value given by the linear-isotherm limit. Finally, to obtain the infinite solubility asymptote, we consider that adsorption is negligible with respect to the bulk concentration, and therefore that this limit is also approximated by the no-flux case. As described above, we solve for concentration at the solid interface and then find the corresponding adsorption equilibrium amount.

The most relevant regions in Fig. $\mathbf{4}$ are those of the dashed and dotted curves: low solubility with weak adsorption and low solubility with strong adsorption. If adsorption is minimal at low solubilities, then wettability alteration is unlikely due to a lack of adsorbed asphaltene molecules. Conversely, large $K / \delta$ allows for maximum surface coverage even with very small solubilities. In this case wettability reversal is more likely. The role of decreasing solubility is clearly demonstrated in Figs. 3 and 4: it increases the equilibration time. For weak adsorption the linear isotherm limit in Eq. 13 emerges, whereas for strong adsorption equilibration time obeys Eq 16.

For these results to be applied to the asphaltene adsorption problem, parameters must be estimated to describe asphaltene behavior in aqueous solutions. Unfortunately, accurate values of $K, \Gamma_{\max }, D$, and $c_{S}$ are difficult to obtain. We choose $D$ and $\Gamma_{\text {max }}$ using Speight's recent review of asphaltene structure ${ }^{15}$. He suggests an average molecular weight of 2000 , based on molecular weight determinations in unaggregating solvents. A proposed monomolecular asphaltene structure is a hydrocarbon sheet of saturated and unsaturated rings. We estimate a reasonable size of $50 \AA$ diameter and $5 \AA$ thick. To determine $D$ we use a theory for diffusion of oblate ellipsoids with radii 2.5 and $25 \AA^{26}$. To be conservative, we use one order magnitude smaller than predicted by the theory, $10^{-7} \mathrm{~cm}^{2} / \mathrm{s}$. An approximate $\Gamma_{\max }$ value of $5 \times 10^{7} \mathrm{~mol} / \mathrm{m}^{2}$ is calculated for a monolayer of these asphaltenes standing on end. A more probable configuration is adsorption with the $50 \AA$ diameter side toward the rock, but again we opt for a conservative estimate by maximizing the adsorption necessary for wettability alteration. Our adopted $\Gamma_{\max }$ value agrees with values found for adsorption from organic solvents onto various mineral oxides ${ }^{13,14,16,17}$. With these two parameters fixed, we consider $K$ ranging from $10^{-9}$ to $10^{-1} \mathrm{~m}$, and $c_{S}$ between 0.1 $\mathrm{ppb}$ and $10^{3} \mathrm{ppm}$.

Fig. 5 is a plot of equilibration time versus solubility for an infinite adsorption constant and for $K=10^{-9} \mathrm{~m}$. As indicated by the dashed line, the small $K$ value corresponds to a linear adsorption isotherm for which equilibration times are independent of solubility. The dotted line provides an upper bound for equilibration time estimation; all finite $K$ values have a corresponding solubility below which equilibration time is independent of solubility. Curves are shown for two water layer thicknesses, $100 \mathrm{~nm}$ and $1 \mu \mathrm{m}$, the latter presumably being the maximum possible thickness of water films existing within a typical pore. For this entire parameter range, the maximum equilibration time is about 10 hours, clearly accessible on laboratory time scales. Every order of magnitude decrease in diffusivity increases equilibration time by one order of magnitude. Even a few orders of magnitude change in parameters maintains the equilibration time, if not within laboratory time scales, certainly well below geological time scales. 


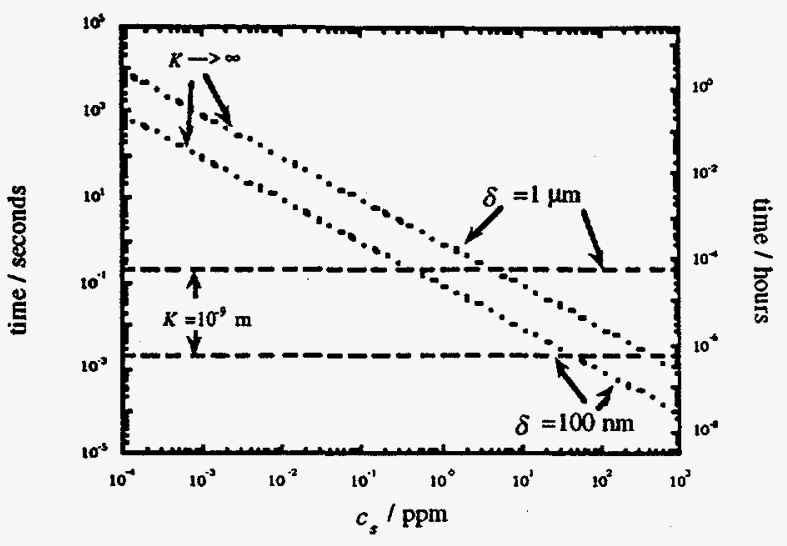

Fig. 5- Adsorption equilibration times as a function of $c_{S}$ for $D=10^{-7} \mathrm{~cm}^{2} / \mathrm{s}$.

\section{Implications}

Our results show that even for extremely low asphaltene solubilities (less than $1 \mathrm{ppb}$ ), equilibrium asphaltene coverage is reached in a few hours. Adoption of unlikely parameter values still demands surface equilibration within laboratory time scales. One effect of low asphaltene solubility is to limit the number of adsorbed molecules that are present at equilibrium; this obviously prevents wettability alteration. However, it is possible to reach saturation coverage with very low solubilities if there is a high adsorption constant. This condition is represented as the $K$ approaching infinity curve of Fig. 5 for very low solubilities, given by Eq. 16 . Equilibration times are still on the order of a few hours, and saturation coverage to $\Gamma_{\max }$ is expected for large enough $K$ (i.e., for $K / \delta \gg C_{S}{ }^{-1}$ ).

Our calculations imply that low solubility asphaltenes can diffuse through water films to arrive at rock surfaces in relatively short time scales. Asphaltene molecules are not prevented from arrival at rock surfaces by a protective water film. Thus, if asphaltene adsorption in the presence of water is strong enough to alter rock wettability, then all asphaltic oil reservoirs should be completely oil wet, which is not observed. We speculate that asphaltic species arriving at the rock surface through water do not adsorb strongly enough to reverse wettability to oil wetness. Apparently, rupture of the water film followed by direct deposition of the asphaltenic material adsorbed at the oil/water boundary is needed to reverse wettability.

Upon water film rupture, the degree of wettability alteration presumably depends on the amount and structure of asphaltenes present at the oil/water interface. Fig. 2 schematically depicts such configurations. As a result of significant asphaltene and resin adsorption, rigid skins may form at crude oil/water interfaces ${ }^{27-29}$, especially when asphaltenes are at the point of incipient flocculation in the crude oil ${ }^{19}$. Deposition of an entire skin suggests strong, irreversible wettability alteration at the locations of water film rupture 4 . While there is evidence that increased aggregate size at the oil/water interface enhances the degree of wettability alteration ${ }^{2}$, the qualitatively essential feature for wettability alteration appears to be direct contact between oil and solid, regardless of the amount and configuration of asphaltenes at the oil/water interface.

\section{Conclusions}

Calculations have been performed of diffusion times for very low solubility asphaltenes from an oil/water interface through a water film followed by adsorption on a rock surface. Contrary to popular belief, our new results show adsorption equilibration within a few hours, and even for extremely low solubilities, within laboratory time scales. Apparently, asphaltene adsorption on reservoir rock in the presence of a finite water film is not strong enough to initiate wettability 
alteration. We argue that water-film rupture followed by direct deposition of crude oil onto rock is the likely origin of wettability reversal in reservoir rock.

\section{Nomenclature}

$a_{i}=$ coefficients in Eq. 11

$a_{v}=$ rock surface area to aqueous film volume ratio

$c=$ asphaltene aqueous concentration

$C=$ dimensionless aqueous asphaltene concentration

$D=$ asphaltene diffusivity in water

$g=$ parameter used in computing coefficients in Eq. 11

$j=$ time step index

$K=$ adsorption equilibrium constant

$m=$ time step index

$n=$ summation index

$t=$ time

$U=$ dimensionless concentration with boundary conditions in Eq. 7

$V=$ dimensionless concentration with boundary conditions defined by Eqs. 6 and 7

$x=$ distance

$z=$ dimensionless distance

$\alpha=$ fraction of surface coverage unadsorbed at equilibrium

$\Gamma=$ adsorption density

$\delta=$ water film thickness

$\zeta=$ dummy integration variable

$\boldsymbol{\theta}=$ surface coverage

$\tau=$ dimensionless time

\section{Subscripts}

$o=$ zero asphaltene adsorption

$e q=$ equilibrium

$\max =$ maximum

$s=$ solubility

\section{Acknowledgments}

This work was supported by the Assistant Secretary for Fossil Energy, Office of Oil, Gas, and Shale Technologies of the U.S. Department of Energy under contract No. DE-AC03-769500098 to the Lawrence Berkeley National Laboratory of the University of California. We thank John Newman for discussion on the asymptotic analyses.

\section{References}

1. Buckley, J. S., and N. R. Morrow: "Characterization of Crude Oil Wetting Behavior by Adhesion Tests," SPE/DOE 20263, proceedings of the 7th SPE/DOE Symposium on Enhanced Oil Recovery, Tulsa, (1990).

2. Buckley, J. S. et al.: "Asphaltenes and Crude Oil Wetting -- The Effect of Oil Composition," SPE/DOE 35366, proceedings of the 10th S.PE/DOE Symposium on Improved Oil Recovery, Tulsa, (1996).

3. Dixit, A. B. et al.: "Pore Scale Modelling of Wettability Effects and Their Influence on Oil Recovery," SPE/DOE 35451, proceedings of the 10th SPE/DOE Symposium on Improved Oil Recovery, Tulsa, (1996).

4. Kovscek, A. R., H. Wong, and C. J. Radke: "A Pore-Level Scenario for the Development of Mixed Wettability in Oil Reservoirs," AIChE J., 39 (1993) 1072.

5. Morrow, N. R.: "Wettability and Its Effect on Oil Recovery," JPT, 42 (1990) 1476.

6. Robin, M., E. Rosenberg, and O. Fassi-Fihri: "Wettability Studies at the Pore Level: A New Approach by Use of Cryo-SEM," SPE Form. Eval., 10 (1995) 11.

7. Salathiel, R. A.: "Oil Recovery by Surface Film Drainage In Mixed-Wettability Rocks," JPT, 25 (1973) 1216.

8. Ball, M. W.: "Athabaska Oil Sands: Apparent Example of Local Origin of Oil," Bulletin of the American Association of Petroleum Geologists, 19 (1935) 153.

9. Melrose, J. C.: "Interpretation of Mixed Wettability States in Reservoir Rocks," SPE 10971, proceedings of the 57th SPE Annual Fall Technical Conference and Exhibition, New Orleans, (1982).

10. Buckley, J. S., K. Takamura, and N. R. Morrow: "Influence of Electrical Surface Charges on the Wetting Properties of Crude Oil," SPE Res. Eng., 4 (1989) 332. 
11. Morrow, N. R., "Introduction to Interfacial Phenomena in Oil Recovery," in Interfacial Phenomena in Oil Recovery, Marcel Dekker, Inc., New York (1991) 1.

12. Denekas, M. O., C. C. Mattax, and G. T. Davis: "Effects of Crude Oil Components on Rock Wettability," Pet. Trans. AlME, 216 (1959) 330.

13. Collins, S. H., and J. C. Melrose: "Adsorption of Asphaltenes and Water on Reservoir Rock Minerals," SPE 11800, proceedings of the SPE International Symposium on Oilfield and Geothermal Chemistry, Denver, (1983).

14. González, G., and A. Middea: "Asphaltenes Adsorption by Quartz and Feldspar," J. Dispersion Science and Technology, 8 (1987) 525.

15. Speight, J. G.: "The Molecular Nature of Petroleum Asphaltenes," Arab. J. Sci. Eng., 19 (1994) 335.

16. Dubey, S. T., and M. H. Waxman: "Asphaltene Adsorption and Desorption From Mineral Surfaces," SPE Res. Eng., 6 (1991) 389.

17. González, G., and M. B. C. Moreira: "The Wettability of Mineral Surfaces Containing Adsorbed Asphaltenes," Colloids Surfaces, 58 (1991) 293.

18. Christenson, H. K., and J. N. Israelachvili: "Direct Measurements of Interactions and Viscosity of Crude Oils in Thin Films between Model Clay Surfaces," J. Colloid Interface Sci., 119 (1987) 194.

19. McLean, J. D., and P. K. Kilpatrick: "Effects of Asphaitene Solvency on Stability of Water-in-Crude Oil Emulsions," J. Colloid Interface Sci., in press (1997).

20. Acevedo, S. et al.: "Interfacial Rheological Studies of Extra-Heavy Crude Oils and Asphaltenes: Role of the Dispersion Effect of Resins in the Adsorption of Asphaltenes at the Interface of Water-in-Crude Oil Emulsions," Colloids Surfaces A, 71 (1993) 65.

21. Defay, R., and G. Petre, "Dynamic Surface Tension," in Surface and Colloid Science, Wiley, New York (1971) 27.

22. Ward, A. F. H., and L. Tordai: "Time-Dependence of Boundary Tensions of Solutions," J. Chem. Phys., 14 (1946) 453.

23. Sutherland, K. L.: "The Kinetics of Adsorption at Liquid Surfaces," Aust. J. Sci. Res., A5 (1952) 683.

24. Hildebrand, F. B.: Advanced Calculus for Applications, Prentice-Hall, Inc., Englewood Cliffs, New Jersey (1962) 451.

25. Carslaw, H. S., and J. C. Jaeger: Conduction of Heat in Solids, Oxford University Press, Oxford (1959) 128.

26. Schmitz, K. S.: An Introduction to Dynanic Light Scattering by Macromolecules, Academic Press, Inc., San Diego (1990) 50.

27. Lawrence, A. S. C., and W. Killner: "Emulsions of Seawater in Admiralty Fuel Oil With Special Reference to Their Demulsilication," J. Inst. Pet, 34 (1948) 821.

28. Bartell, F. E., and D. O. Neiderhauser: "Film Forming Constituents of Crude Petroleum Oils," Fundanental Research on Occurrence and Recovery of Petroleum, 1946-47, API, (1949) 57.

29. Denekas., M. O. et al.: "Materials Adsorbed at Crude Petroleum-Water Interfaces," Ind. Eng. Chem., 43 (1951) 1165. 